\title{
Hemorragia cerebelosa y edema pulmonar neurogénico
}

\author{
Dr. Iván Casas C. 1 ; Dra. Marisd del Valle M. 1 : \\ Dr. Jorge Foster M. ${ }^{2}$; Dra. Eva Nilsen V. 3 .
}

\section{Cerebellar hemorrhage and neurogenic puhmonary edema}

\begin{abstract}
A twelve year old male patient with an spontaneous cerebellar hemorrhage (S.C.H.) and neurogenic pulmonary edema (N.P.E.) is reported. On admission he was in coma and died six hours later. Both S.C.H. and N.P.E. are uncommon in children. S.C.H. may present with an acute or chronic course: its nost frecuent symptoms are headache, nausea, vomiting, ataxic gait, cranial nerve irvolvement, altered consciousness and brainstem disfunction. N.P.E. presents with respiratory distress, cyanosis, pink-tinged sputum, rales and tachycardia. S.C.H. and N.P.E. may be fatal, so a prompt diagnostic and therapeutic approach is vital. The most important characteristics of these two entities are discussed.
\end{abstract}

(Key words: neurogenic pulmonary edema, spontaneous cerebellar hemorrhage).

Los accidentes vasculares cerebelosos se encuentran con frecuencia en recién nacidos de bajo peso ${ }^{1.2}$, sin embargo, son raros en escolares y adolescentes, habiéndose reportado no más de 50 casos en estos grupos etáreos en la literatura. Su curso es variable, pudiendo ser agudo/crónico, y sus manifestaciones clinicas más comunes son: cefalea, náuseas, vómitos, alteraciones de la marcha. trastornos de la función de los pares craneanos, compromiso sensorial y disfunción troncoencefálica. Es difícil difetenciarlos de otros tipos de hemorragia intracraneana. La etiología más frecuente corresponde a malformaciones vasculares, aunque en un número importante de casos no es pusible establecer la causa de la hemorragia ${ }^{3}$. Las discrasias sanguíneas, los traumatismos, las infecciones y los tumores se encuentran rara ve $z$ dando origen a accidentes vasculares cerebelosos. El diagnóstico se basa en la sospecha clinica, y puede ser comprobado mediante tomografía computarizada. El tratamiento quirúrgico oportuno puede ser en algunos pacientes la única alternativa de sobrevida, pero en algunos casos, como el del paciente que a continuación se describe, el curso es muy rápido y las complicaciones pueden llevar al desenlace fatal en pocas horas sin permitir su tratamiento quirúrgico.
1. Becados, Depto. de Pediatría, Universidad Católica de Chile.

2. Médico Neurología Infantil, Ho spital Sótero del Rio.

3. Médico Anatomía Patológica, Hospital Sótero del Rio.

\section{Caso clínico}

Paciente de 12 años, de sexo masculino, desarrollo pondoestatural adecuado, sin antecedentes mórbidos de importancia. Estando en 
buenas condiciones, cayó bruscamente al suelo. Al levantarsc, se quejó de cefalea holocránea intensa, agregándose después vómitos, sudoración y tendencia al sueño. Mientras era conducido a servicio de urgencia presentó rigidez de las extremidades y desviación de la mirada hacia arriba.

Ingresó treinta minutos después de la caida, en coma, con anisocoria por midriasis izquierda. respiración superficial, frecuencia respiratoria (FR) $64 x$ min $y$ presión arterial (PA) $200 / 130 \mathrm{~mm}$ de $\mathrm{Hg}$. Se le administró oxígeno y $20 \mathrm{mg}$ de furosemida endovenosa, inmediat amente después presentó paro respiratorio, siendo necesario cl uso de ventilación mecánica, bicar bonato de sodio, gluconato de calcio y fenobarbj. tal. Treinta minutos después, su frecuencia cardíaca era de $140 \times \mathrm{min}$. la presión arterial $130 / 100 \mathrm{~mm}$ de $\mathrm{Hg}$ y la temperatura axilur de $35^{\circ} \mathrm{C}$. Evolucionó en coma, con sinergia de descerebración ante estímulos nociceptivos, reflejos osteotendíneos y cutaneos abdominales nomnales, respuesta plantar flexora, miosis y ausencia de reflejos pupilares. No se detectaron signos de irritación meningea en ningún momen to de la evolución. En el fondo de ojo habian hemorragias retinianas subhialoideas bilaterales.

Al ingresar, glicemia, kalemia y natremia eran normales. Mientras el paciente era ventilado manualmente se registraron cil la sangre arterial $\mathrm{pH} 7,32 . \mathrm{PO}_{2} 59,4 \mathrm{~mm} \mathrm{Hg} . \mathrm{PCO}_{2} 31,7 \mathrm{~mm} \mathrm{Hg} \mathrm{y}$ exceso de base (BE)-4.

Tres horas después de ventilación mecánica con presión inspiratoria máxima (PIM) de $26 \mathrm{~cm}$ de agua, FR de 26 por minuto y concentración de $\mathrm{O}_{2}$ en el aire inspirado $\left(\mathrm{FiO}_{2}\right)$ de $70 \%$, se obtiene pH 7.66. $\mathrm{PO}_{2} 26 \mathrm{lmm} \mathrm{Hg}, \mathrm{PCO}_{2} 11 \mathrm{~mm}$ $\mathrm{Hg}$ y $\mathrm{BE}--0.8$
Posteriormente aparecieron, sucesivamente, hipertermia, poliuria y taquicardia que se mantuvieron en las tres horas siguientes, luego midriasis paralítica bilateral, hipotensión arterial. extrasístoles ventriculares, bradicardia, Ilene capilar lento, anuria y paro cardiaco, falleciendo seis horas después del ingreso. La punción lumbar post mortem, dio salida a líquido céfalo raquideo hemorrágico que no coagulaba

En el estudio anátomo patológico el cráneo ara de forma normal, sin lesiones aparentes. E1 cerebro estaba muy tumefacto: pesaba $1.440 \mathrm{~g}$ (nomal 1.350), tenía gran hiperemia de los vasos de las leptomeninges. dilatación y hemorragia intraventricular bilateral (fig. 1). Los vasos del polígono de Willis eran de aspecto normal. El cerebelo estaba muy tumefacto y de él salia espontáneamente sangre y coágulos. Las amigda. las cerebclosas hacian hernia, observándose una gran hemorragia en el hemisferio cerebeloso izquierdo con destrucción del parénquima, sin evidencias de tumor $\mathbf{n i}$ de vasos aberrantes (fig. 2). E] ex:men histológico mostró edema cerebral, necrcisis de colicuación y focos de hemorragia. La tráquea y los bronquios se vejan inundados por un líquido serohemático. Cada pulmón pesaba $470 \mathrm{~g}$ (normal 190 a $200 \mathrm{~g}$ ) sus serosas eran lisas y brillantes; los lóbulos supe. riores se veían rosados, mientras el lóbulo medio y los inferiores eran de color rojo oscuro. A! corte, el parénquima tenia aspecto hemorrágico. y el estudio histológico mostró edema intraalveolar e intrabronquial importante, con extensas áreas de hemorragia. El higado y el bazo tenían mayor peso que el habitual y evidencia de hiperemia pasiva. En los riñones no se encontraron alteraciones histológicas

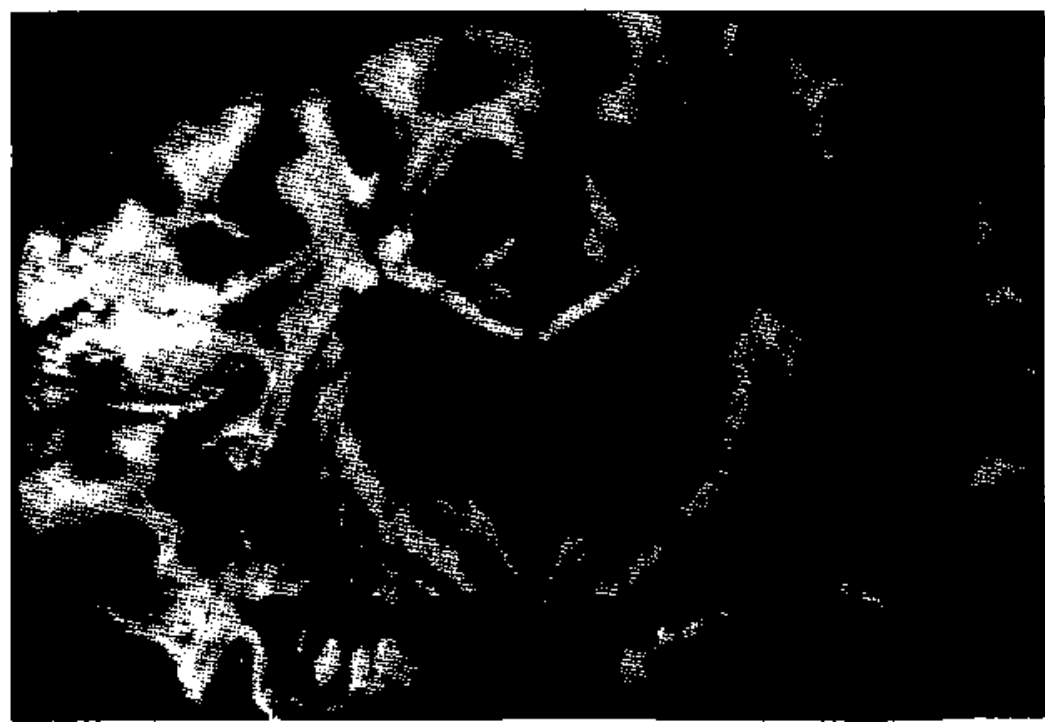

Fig. 1. Sistema ventricular dilatado e inundado con sangre. 


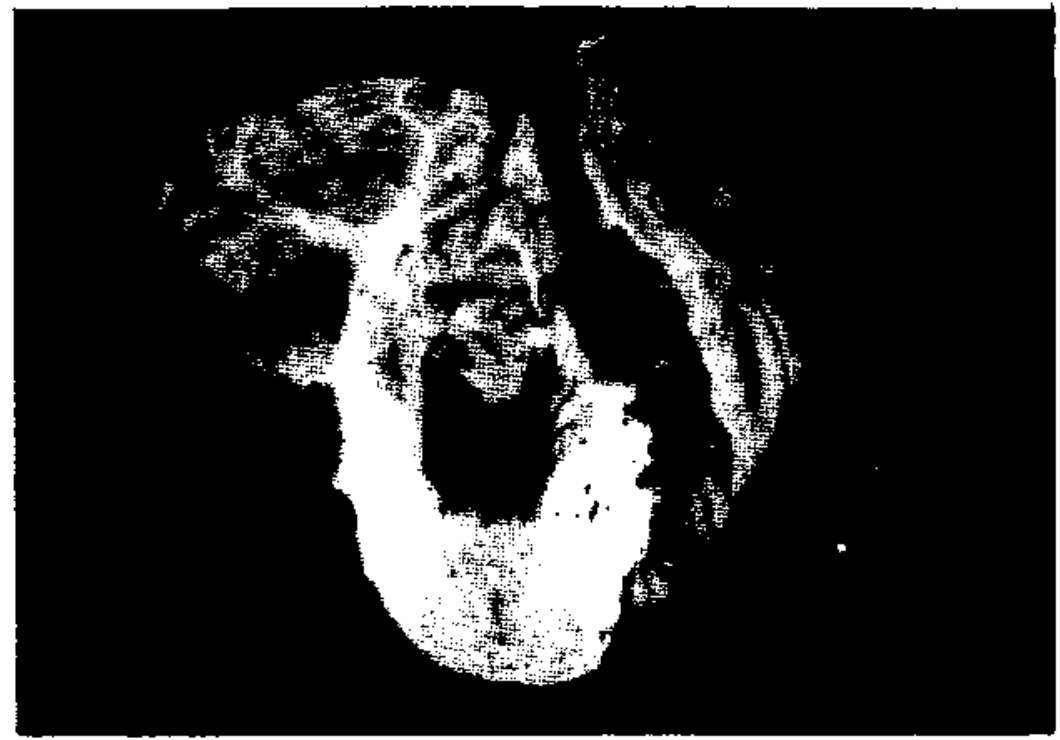

Figura 2. Hemorragia intraparenquimatosa de hemisterio izquierdo, abierta a sistema ventricular.

\section{COMENTARIO}

La hemorragia cerebelosa es poco frecuente en niños. correspondiendo al $10 \%$ de las hemorragiàs no traumáticas 5,7 .

En el período neonatal, la hipoxia parece ser la primera causa de hemorragia cerebelosa. En un porcentaje no despreciable de niñus mayores no se encuentra etiología; en los que se demuestrá causa, ésta corresponde fundamentalmente a malformaciones vasculares (62\%), siendo mucho menos frecuentes las alteraciones hematológicas, los tumores sólidos, las infecciones y los traumatismos $3-6,9,10$

Las manifestaciones clínicas de la hemorragia cerebelosa son muy variables; muchas veces se trata de pacientes aparentemente sanos que en forma brusca presentan vómitos, compromiso de conciencia y signos neurológicos caracteristicos de un proceso expansivo de fosa posterior. Plum y Foster ${ }^{11}$ dividen a los pacientes en tres grupos según sus manifestaciones clínicas, evolución y pronóstico. El primer grupo incluye a los que presentan pérdida de conciencia brusca, irregularidad en el ritmo respiratorio, miosis $y$ ausencia de reflejos óculo vestibulares. Evolucionan en forma tórpida y generalmente fallecen. El segundo grupo está constituido por aquellos pacientes que presentan cefalea occipital, náuseas, vómitos, vértigo, debilidad de extremidades inferiores y desviación ocular tónica. Evolucionan con deterioro neurológico progresivo, esta. bleciéndose el sindrome completo en horas o días. Esta es la forma de presentación más frecuente de hemorragia cerebelosa y su pronóstico depende del diagnóstico oportuno y tratamiento adecuado. Los pacientes del tercer grupo se caracterizan por presentar cefalea occipital $y$ alteraciones sensoriales más o menos progresivas: o disfunción cerebelosa u oculomotora progresiva, evolucionan en forma favorable, pudiendo recuperarse en forma espontánea.

Cuatro aspectos de la patogénesis explican los sintomas de este grupo de pacientes: las hemorragias cerebelosas pueden producir destrucción total del parénquima $y$, secundariamente, por efecto de su masa, aumento brusco de la presión en la fosa posterior, fenómeno responsable de la formación de hernias cerebelosas. La dislocación del cerebelo puede producirse hacia arriba, a través del tentorio, provocando compresión dorsal, en el agujero occipital, del bulbo raquideo. Puede ocurrir también que la hemorragia se abra paso hacia el cuarto ventriculo, produciéndose gran aumento de la presión intraventricular y severa alteración de la función del troncoencéfalo. Por último, se puede obstruir el flujo de líquido cefalorraquídeo, desarrolländose hidrocefalia.

Los mecanismos descritos pueden ocurrir juntos o aislados, determinando el curso clinico del paciente

Un alto índice de sospecha clínica es lo más importante para reconocer la hemorragia cerebelosa: la punción lumbar está contraindicada cuando se sospecha una masa de fosa posterior, por la posibilidad de proyocar hernias cerebelosas $^{12}$. La ecoencefalografía y la neumoencefalografía tienen un valor limitado, consumen un tíempo valioso $y$ pueden ser peligrosos ${ }^{3}$. El 
diagnóstico actualmente puede ser confirmado mediante tomografía axial computarizada. La arteriografía cerebral suele ayudar en la identificación de la alteración vascular de origen ${ }^{3}$.

E1 tratamiento de los pacientes con hemorragia cerebelosa es operatorio: la indicación de exploración quirúrgica de la fosa posterior es clara en niños que evolucionan con deterioro progresivo. Aunque es posible la recuperación espontánea de algunos pacientes, este heclıo no ha sido publicado en la literatura ${ }^{3.1} 1$

El edema pulmonar neurogénico (EPN) es un problema poco descrito en niños. generalmente se asocia con status convulsivo ${ }^{13}$. Clinicamente se manifjesta por dificultad respiratoria, cianosis, expectoración hemoptoica, estertores pulmonares difusos, taquicardia, fiebre moderada, infiltrados de relleno alveolar en las radiografías de tórax. hipoxemia y leve leucocitosis. Siempre es necesario descartar una causa cardiaca. Cuando es severo, la muerte sobreviene en horas. Se crce que la agresión del sistema nervioso central alteraría la dinámica de los capilares pulmonares provocando cambios en la presión y permeabilidad vascular del pulmón. Estos cambios serían mediados por efectos alfa adrenérgicos. beta adrenérgicos y vagales, probablemente ocasionados por el gran aumento de la presion intracra. neana, hecho común en todas las situaciones en que ocurre edema pulmonar neurogénico. El dumento de la presión intracraneana actuaria sobre ciertas zonas del sistema nervioso central (probablemente bulbares). las descargarían los mediadores responsables de lat alteración del lccho vascular pulmonar.

El tratamiento del edema pulmonar neurogénico incluye el cmpleo de ventilación mecánica con aplicación de presión positiva al final de la espiración y control directo de la presión intracraneana. Aún no se ha evaluado cl uso profiláctico ni terapéutico do agentes alfa bloqueado$\operatorname{res}^{14,15,16}$

\section{RESUMEN}

Se presenta el caso clinico de un niño de 12 años con hemorragia cerebclosa y edema pulmonar neurogénico que ingresó en coma y falleció al cabo de seis horas. La afección basal y la cumplicación descritas son raras en niños; la hemorragia cercbelosa se caracteriza por manifestaciones clinicas variables, agudas a crónicass, siendo los sintomas más frecuentes cefalea, náuseas, vómi- tos, alteraciones de la marcha, trastornos de la función de los pares craneanos, compromiso sensorial y disfunción troncoencelalica. Las manifestaciones clinicas del edema pulmonar neurogé. nico son dificultad respiratoria, cianosis, expectoración hemoptica, estertores puimonares difuses y taquicardia, La hemorragia cerebclosa pucde ser fatal y tienen gran importancia el diagnóstico precoz y tratamiento uportuno médico y quirúrgico.

\section{REFERENCIAS}

1. Grunnet, M.L., Shields. W.D.: Cerebellar llentorrhage in the Prenisture Infant J Pediatr 88: 605. 1976.

2. Martin, R., Resmann, $U$, Fanaroff, A.: Masive intracerebellar hemotrlage in low birth wcight infunts. J Pediatr 89: 290, 1976.

3. Kneeland, W.F.: Spontancous Cerebellar Hemorihage in Children and Adolescents. Am J Dis Child 135: 167,1981

4. Fernández-Alvarez, E., Fabregues, I., Pinedo, M. Costa, J., Lafuense, J.: Post traumatic Cerebellar I Iematoma. Child's Brain 9: 362, 1982.

5. Mc Kissock, W., Richardson, A., Walsh. L..: Spontaneous Cerchellar Henlorrhage: A study of 34 consecutive cases trated surgicalley. Baain 83: 1, 1960 .

6. Vincent, F.M. Bartone. J.R., Jones, M.Z.: Cerebellar astrocy toma presenting as cerebe]lar hemorrhage in a cluild. Neurology 30:91, 1980.

7. Ott, K.H., Kase, C.S., Ojeman, R.G., Mohr, J.P.: Cerebellar Hemorthage: Diagnosis and Treatment. Arch Neurol 31: 160, 1974.

8. Eremberg, G., Rubin, R., Sthulman, K.: Cerebellar haematomas caused by angiomas in children. J Neurol Neurosurg Psychiatry 35: 304, 1972.

9. Kazminoff, P.B., Weichsel, M.E., Grinnell, V., Young R.F.: Acute cerebellar hemorrhatge in Childhoud: Ftiology, Diagnosis and Treatment. Neurosurg 6: $524,1980$.

10. Marlin, A.: Cetebellar Vascular Accidents in Children. Concept in Pedtatric Neurosurgery 3. Editado por Raimondi A. Editorial Karger, Chicago: 75,1973 .

11. Plum, F., Posner, J.: Subtentorial lesions causing coma. The diagnosis of stupor and coma. Editado por F.A. Davis Company, Philadelphia: 120, 1972.

12. Swaman, $K$., Wright, $F_{-}$: The practice of pediatric Neurology. The C.V. Mosby Company, 1982.

13. Mulroy, J.J., Mickell, JJ., Tong, T.K., Pellock, J.M.: Postictal pulmonay cdema in chiden. Neurology $35: 403,1985$

14. Theodore, J., Robin, E.D.: Speculations on Neuro genenic Pumonay Edema. An Rev Resp bis 113 $405,1976$.

15. Colice. G., Matthay, M., Bass. E, Natthay, $R$. Neurogenic Pulmonary Ldema. Am Rev Resp Dis 130: 941,1984

16. Wauchob. T., Broocks, R., Harrison. K.: Neurogenic Pulmonary Eđema. Anesthesia 39: 529, 1984. 